\title{
Tuning structural parameters for the optimization of drug delivery performance of cyclodextrin-based nanosponges
}

VENUTI, V., ROSSI, B., MELE, ANDREA, MELONE, LUCIO, PUNTA, CARLO, MAJOLINO, D., MASCIOVECCHIO, C., CALDERA, F., TROTTA, F.

\section{Highlights box}

- The current status of a wide characterization of structural and dynamical properties of cyclodextrin nanospones, by complementary techniques, is here reported.

- The complex interplay between covalent and non-covalent interactions occurring in the cyclodextrin units within the polymer is thought to be responsible of the molecular architecture and rigidity of the entire polymer network.

- A model describing the swelling behaviour of cyclodextrin nanosponges, leading to the formation of hydrogels, is proposed. It is consistent with the experimentally observed transition, for these systems, from highly viscous gel to liquid phase, as a function of hydration level, temperature, and $\mathrm{pH}$.

- A separate investigation of the molecular dynamics of confined water and nanosponge polymer matrix, thanks to the complementarity of Raman and FTIR-ATR spectroscopies, allowed a full understanding of the molecular mechanisms regulating the macroscopic response of hydrogels.

- This currently achieved knowledge represents a crucial prerequisite for the rational design of cyclodextrin-based hydrogels having specific performances for controlled release of bioactive ingredients, as discussed.

- The case study concerning transport phenomena of a model drug, i.e. ibuprofen sodium salt, as investigated by (HR-MAS) NMR spectroscopy, is reported. 


\begin{abstract}
Introduction In view of a development of new soft materials, nanostructured self-assembled systems have recently attracted great attention in a variety of technological fields of high social impact. Cyclodextrin nanosponges (CDNS) represent a new and highly versatile class of crosslinked cyclodextrin (CD)-based nanoporous polymers. Their intriguing properties, including safety, biodegradability, negligible toxicity, marked swelling behavior, superior inclusion capability with respect to native $\mathrm{CD}$, are the bases for potential for applications in drug delivery, tissue engineering and regenerative medicine.
\end{abstract}

Areas covered In the current review, we report on the state-of-art concerning a detailed characterization of structural and dynamical features of CDNS explored by the combined use of different and complementary techniques, such as Fourier transform infrared absorption in attenuated total reflectance geometry (FTIR-ATR) and Raman spectroscopies, and high resolution magic angle spinning (HR-MAS) NMR spectroscopy. The ambitious objective is to furnish an exhaustive survey of the role played by hydrophobic and hydrophilic groups within the cross-linked network, in dry and swollen state, in determining the macroscopic functional features of CDNS.

Expert opinion The reported results can significantly contribute in the rational design and optimization of new stimuli-responsive systems exhibiting tuneable inclusion/release properties, adapted to the therapeutic demands of pathology.

\title{
Keywords
}

Cyclodextrin nanosponges, FTIR-ATR spectroscopy, Hydrogel, Raman spectroscopy, HR-MAS NMR spectroscopy. 


\section{Introduction}

Nowadays, the application of nanostructures and nanoparticles in medicine as targeted and sustained drug delivery systems (nanomedicine) represents an actual alternative to standard therapy, allowing to potentially address two medical challenges of conventional medicine, namely the poor selectivity and the high toxicity of classical protocols [1,2]. The promising benefit of the nanomedicine approach pushes towards the design of novel, bio-inspired nanocarriers and nanostructured biomaterials, and to the in-depth investigation of their nanoscale structural characteristics.

In this context, cyclodextrin-based nanosponges (CDNS) have attracted an increased interest in the last two decades, due to their high versatility as nanocarriers, combined with very simple and cheap synthetic protocols. CDNS are innovative three-dimensional polymers obtained from monomeric cyclodextrin (CD) by chemical cross-linking [3-5]. The micro- and nanoporosity resulting from the cross-linking reaction reveals the potential of CDNS both as sorbent and releasing material. These polymers can be obtained by a step-growth polymerization of native CD with a suitable crosslinking agent. According to the chemical nature of the cross-linking used, some properties of the polymer can be predicted and modulated, such as the swelling capability, the electric potential and the balance between the hydrophilic and hydrophobic parts.

DeQuan Li and Min Ma were the first to introduce the term "cyclodextrin nanosponge" in 1998 to describe $\beta$-cyclodextrin units cross-linked with organic diisocyanates [6], even if insoluble crosslinked CD-polymers had been already described in the literature. The resulting insoluble network was successfully proposed for water decontamination from different organic pollutants [7]. However, no other applications were claimed, until the syntheses of new families of CDNS revealed their full potential for pharmaceutical applications as drug carriers [8-11].

Tejashri et al. recently reported a comprehensive overview on the methods of CDNS preparation and on the wide range of cross-linkers used for this purpose [12].

Briefly, the standard protocol consists into the reaction of the primary hydroxyl groups of the Dglucopyranose units of CDs (the monomers) with activated cross-linkers (e.g. harmless synthetic equivalents of phosgene to afford carbonates, vide ultra). The reaction can occur under solvent-free conditions, following the so called "melt protocol" at $100{ }^{\circ} \mathrm{C}$, or in the presence of a suitable aprotic polar solvent (DMF or DMSO), at temperatures ranging from $10{ }^{\circ} \mathrm{C}$ to reflux. With the latter approach, the cross-linked polymer can jellify directly from the reaction medium. A third alternative protocol consists of ultrasonication of CDs and cross-linkers in the absence of solvent [13]. Regardless of the protocol of choice, the final product needs to be washed several times with suitable solvents (preferably water) to remove unreacted starting materials and by-products. Figure 
1 schematically describes the different cross-linkers suitable for CDNS synthesis. Besides the original diisocyanates, such as hexamethylene diisocyanate (HDI) and toluene-2,4-diisocyanates (TDI), active carbonyl compounds, including carbonyldiimidazole (CDI), diphenyl carbonate (DPC), and trifosgene, have been also widely used, leading to the formation of carbonate-CDNS with enhanced capability to encapsulate a wide range of pharmaceuticals [14].

An even simpler and faster protocol can be followed by using dianhydrides as crosslinking agents, in the presence of an organic base, following a classical step-growth polymerization protocol. Pyromellitic dianhydride (PMA) or the activated form of ethylenediaminetetraacetic acid (EDTA) afford the nanostructured polymer by jellification from DMSO within a few minutes. These networks exhibit interesting swelling properties, promoting the formation of hydrogels, whose drugdelivery efficiency can be easily tuned (see section 3 ).

CDNS can form complexes with different types of molecules, resulting particularly suitable for improving the solubility of hydrophobic molecules in aqueous medium and protecting degradable substances $[10,11]$. The loading may be realised by directly adding drugs, solubilized in suitable volatile solvents or in water, to the CDNS powder or by mechanically mixing the CD-polymer with the target molecule.

The release kinetics of the entrapped molecules can be modulated by modifying the reaction conditions and, consequently, the final nanostructured network. As a consequence, CDNS were reported to deliver the drug to the target site even three to five times more effectively than direct injection [15].

These materials are generally safe and biodegradable, and show a negligible toxicity also confirmed in vivo by direct administration in mice. [16]

CDNS result in a solid partially crystalline or non-crystalline powder, depending respectively on the melt or solvent method followed during the synthesis. The resulting powder can be easily formulated for oral administration, by dispersion in a suitable matrix, and in parenteral and topical dosage forms, by preparing aqueous solutions or relatively stable hydrogels $[17,18]$.

Moreover, CDNS are also efficient carriers for proteins and other macromolecules, such as enzymes, DNA and oligonucleotides, to be used in drug and gene therapy [19]. The administration of these molecules is usually a challenge. In fact, the high molecular weight and the hydrophilic character, also associated to a high surface charge, determine a low affinity with the lipophilic biological membranes, limiting the possibility of cellular internalization. Following intravenous administration, drug degradation can rapidly occur. With oral administration, bioavailability becomes an issue. In order to overcome these limits, conventional therapy usually suggests to increase the dose or to combine the macromolecule with absorption promoters. However, both these 
approaches increase the level of risk related to toxicity. The adsorption and encapsulation of proteins and other macromolecules in CDNS provides a new class of nanocarriers, overcoming the limits above described. In the case of CDNS-encapsulated enzymes, their activity and efficiency are even enhanced in terms operative temperature and $\mathrm{pH}$ range.

CDNS have been also proposed as efficient carriers for gases like $\mathrm{O}_{2}$ and $\mathrm{CO}_{2}$, resulting attractive for many other biomedical applications. In particular, the $\mathrm{O}_{2}$-nanocarrier CDNS nanosponges could provide oxygen to cure hypoxic tissues usually associated to various diseases [20].

Finally, the same nanocarriers can selectively absorb biomarkers, playing the role of nanoprobes for diagnostic medicine [21]. Thus, CDNS can combine in an unique solution two complementary technologies, nanodiagnostics and nanoparticle-mediated drug delivery, proposing themselves as ideal candidates for "theranostic" technology, the new frontier of nanomedicine.

Even if the advantage of using CDNS as efficient nanocarriers is by now well documented, the potentiality of these systems is far to be completely disclosed. Several parameters in the synthetic process, including the type and concentration of cross-linker, the dimension of the cyclic monomer $(\alpha, \beta$ or $\gamma-C D)$, and the cross-linking degree, can influence the nanostructure and nanoporosity of the network, tuning the efficiency of drug diffusion in the carrier and of drug release in biological environment. A complete comprehension of the effect of these variables onto the final network passes through the characterization of structural and dynamical features of CDNS.

As is well known [22], IR and Raman spectroscopic methods represent a very useful tool for the structural elucidation of molecular systems and can provide, in several cases, complementary information due to their different selection rules. Symmetric vibrations and ring breathing modes are generally strong in Raman scattering, but forbidden (or, in any case, weaker) in the IR spectra; on the other hand, several asymmetric vibrations along the bonds with a permanent (and strong) dipole moment are more easily detected in IR than in Raman spectroscopy. It is important to remind here that the main parameters, i.e. centre-frequencies and intensities, are generally strongly dependent on the overall chemical and physical properties of the molecule under investigation, well expressed in the so-called "fingerprint"' region. A detailed band assignment thus requires massive use of quantum chemical calculations aimed at reproducing both IR/Raman vibrational frequencies and intensities. In our case, the simultaneous use of IR and Raman techniques revealed first of all successful for the study of the complex polymeric network of cyclodextrin nanosponges [23-27]. Again, in the case of hydrated systems, vibrational spectra are particularly informative of the intraand intermolecular modes of water molecules whose oscillator forces are sensitive, in turn, to the interactions with the solute or in general with their surroundings. In this sense, the inspection of Raman and infrared spectra of pure water and water-mixtures constitutes an indirect way of 
studying the hydrogen bond (HB) intermolecular network of water molecules and their different levels of connectivity [24-27].

\section{CDNS in the dry state}

Despite of all the aforementioned possibilities of application, the comprehensive characterization of dry CDNS in the solid state, involving both structural and dynamical points of view, has been limited by some factors: the random nature of the growing process of the polymer, the high number of reaction sites present on each $\mathrm{CD}$ unit, and the tendency of $\mathrm{CD}$ to give rise to amorphous polymers. New hyper-reticulated polymers have been obtained by co-polymerization of cyclodextrin and calixarene units (CD-Cn copolymers) and proposed [28] as a new class of nanosponges. Their characterization relies upon FT-IR, thermogravimetric, ${ }^{13} \mathrm{C}\left\{{ }^{1} \mathrm{H}\right\}$ CP-MAS NMR and nitrogen adsorption/desorption techniques.

A thorough characterization at molecular level of these systems in the absence of solvent is an essential starting point for the design of the applications of the materials. The spatial disposition of the functional groups and the conformational properties of the cross-linked network, as well as the polymer dynamics of CDNS, play a key-role in determining their macroscopic functionality. Indeed, the search for a clear-cut relationship between polymer structure/morphology and some key properties, such as the water retention capability or the inclusion/release properties, is still a challenging task. A convenient and fruitful strategy consists in the use of vibrationl spectroscopies, FT-IR and Raman, both individually and in a joint and complementary way. In this context, an FTIR study concerning drug loading in nanosponges was performed by Ansari et al. [29], revealing, upon complexation, minor changes in the fingerprint region (i.e. 900-1400 $\mathrm{cm}^{-1}$ ) of the FT-IR spectrum of resveratrol. Again, A. Singireddy et al. [30] used FT-IR and Raman spectroscopy, along with other techniques, to characterize the inclusion complexes of quercetin with CDNS, confirming the interaction between drug and nanosponges.

In the last few years, we applied a multi-methodological approach consisting in the complementary use of FT-IR spectroscopy in attenuated total reflectance (FTIR-ATR) geometry, Raman scattering techinques, and quantum-chemical calculations. We applied this strategy to the study of different classes of ester-bridged $\beta$-CDNS in the dry state [23-25, 27, 31, 32]. The aim was an investigation, at molecular scale, in a wide spatial-temporal window, of the structure and dynamics of these polymers by varying some of the parameters characteristic of the synthesis.

The complex interplay established between non-covalent bonds, i.e. hydrogen bonds (HB) connecting the $\mathrm{CD}$ units within the polymer network, and covalent interactions, turned out to be crucial in determining the molecular architecture and rigidity of the polymeric matrix. More 
specifically, it has been put into evidence how changes in the type of monomeric $C D$, in the chemical structure of the cross-linker, and in the cross-linking agent/CD molar ratio $n$ [23, 27], result in modifications of the reticulation degree of the CDNS network and stiffness of the material itself.

In particular, the evolution of IR/Raman intensities of the spectral sub-bands assigned to the $\mathrm{C}=\mathrm{O}$ stretching vibrational modes of carboxylic and ester groups - typically falling in the $1500-1800 \mathrm{~cm}^{-}$ ${ }^{1}$ wavenumber range - was monitored as a function of $n$ in a collection of CDNS obtained from PMA. The ester contribution was found to grow with respect to $\mathrm{COOH}$ up to a 6-fold excess of cross-linker with respect to $\mathrm{CD}$. For $n$ values higher than 6 , a branching of CD units, rather than reticulation, has been reasonably hypothesized as a consequence of steric crowding, as indicated by the increasing contribution of $\mathrm{COOH}$ with respect to the ester. Thus, quite fundamental information on the type of connectivity in these materials could be derived.

We also inspected the low-wavenumber Raman spectral features of these CD-based polymers [27], with particular regard to the so-called Boson peak (BP), typical of amorphous materials [33-41], strictly related to the elastic properties of the system on a mesoscopic length scale. As a rule of thumb, the position of BP maximum shifts towards higher energies when the stiffness of the systems increases.

By monitoring the $n$-dependence of the stiffness of the polymeric network for PMA- and EDTAbased nanosponges, a similar trend was found, recalling the one observed for the connectivity, exhibiting a maximum of the rigidity in correspondence of a six-fold excess of cross-linker with respect to the monomer $\beta-\mathrm{CD}$.

[Please insert Figure 2 about here]

Finally, the analysis of the high-frequency window of IR/Raman vibrational spectra allowed us to study the effect of the temperature and the $n$ cross-linker/ $\beta-\mathrm{CD}$ molar ratio on the H-bonded network involving the $\mathrm{OH}$ groups of the polymers [24, 25, 31].

As a conclusion of this section, the unprecedented results obtained by this structural and dynamic characterization of this model class of nanoporous materials suggest that the experimentalnumerical here illustrated can be proposed as a promising tool other amorphous polymers for which X-ray diffraction methods cannot be applied because of the low degree of crystallinity.

\section{CDNS in the hydrated state: hydrogel formation}

CD-based hydrogels have been firstly reported by J. Li et al. [42], and, in the following, a variety of studies can be found in the literature mainly regarding hydrogels formed by threading $\alpha-\mathrm{CD}$ rings onto PEG-grafted copolymers [43-48]. It was demonstrated that these undergo gel-sol transition at a 
temperature depending on both polymer structure and chemical composition. A molecular characterization by FT-IR spectroscopy was proposed by Karim et al. [49] in the case of a hydrogel made of a tri-component copolymer PLLA/DMAEMA/PEGMA and $\alpha-C D$.

CDNS have been proved to exhibit a marked swelling ability when contacted with water or liquid solutions, affording hydrogels with intriguing properties [24-26]. These polymers are able to retain large amounts of water, depending on the chemical characteristics of the matrix, making CDNS hydrogels very interesting for applications as scaffolds for drug delivery and, potentially, as innovative soft materials for tissue engineering and regenerative medicine [27,50,51]. The knowledge, at molecular level, of the structural features of CDNS hydrogels represents a pivotal prerequisite in view of a rational design of new smart hydrogels with tunable inclusion/release properties.

An important aspect concerns the changes observed in the properties of CDNS hydrogels by varying the relative amount of water with respect to the polymer component, i.e. the hydration level $h$ (defined as weight ratio $\mathrm{H}_{2} \mathrm{O} / \mathrm{CDNS}$ used in the preparation of the hydrogel). It was shown that CDNS hydrogels tend to evolve from gel state to liquid state by a gradual increase of the hydration level [52]. This behaviour, exhibited by all of the CDNS hydrogels examined, was investigated mainly by the analysis of the spectral changes observed in Raman and FTIR-ATR spectra as a function of $n$. The interpretation of the experimental data led to the conclusion that the maximum water holding capacity and the viscosity of the gel phase are determined by the interplay between physical and covalent interactions that combine over different length-scale to yield the formation and stabilization of the hydrogel network [53].

A semi-quantitative parameter that describes the water-adsorption capacity in different CDNS can be identified in the so called cross-over hydration level $h_{\text {cross }}$, defined and experimentally estimated for all of the CDNS hydrogel investigated. This quantity identifies the cross-over between two different populations of water molecules entrapped in the hydrogel matrix: the populations arranged in "ordered" and "non-ordered" hydrogen bond (HB) connectivity pattern, respectively. The relative amount of the two different populations of water molecules can be estimated by looking at the modifications occurring in the $\mathrm{O}-\mathrm{H}$ stretching and $\mathrm{H}-\mathrm{O}-\mathrm{H}$ bending profiles of water in the vibrational spectra of CDNS hydrogels. Indeed, the tetrahedral connectivity pattern of $\mathrm{H}_{2} \mathrm{O}$ becomes favoured with respect to the non bulk-like arrangements of the water molecules for $h>h_{\text {cross }}$. More interestingly, the cross-over hydration level seems to match also with the value of hydration above which the system evolves from a rigid gel state to a fluid suspension [50-52].

The mass-ratio parameter $m$, defined as $m=1 / h_{\text {cross }}$, can be used for a quantitative measurement of the maximum water holding capability in CDNS. This quantity can be correlated to the molar ratio 
$n$ that was in turn connected to the evolution the elasticity and cross-linking density in CDNS polymers.

[Please insert Figure 3 about here]

The complementarity of FT-IR and Raman vibrational spectroscopies has been exploited to describe the contributions of the water molecules and the polymer backbone to the structural and dynamic features of CDNS hydrogels. [54-56]. Indeed, it was shown that FTIR-ATR spectroscopy is the election method to investigate the molecular connectivity and the extent of HB patterns of water engaged in CDNS hydrogels network. The combined analysis of the vibrational spectra of the polymers hydrated with water and deuterated water allowed us to separate the $\mathrm{HOH}$ bending mode of entrapped water molecules from the vibrational modes related to the $\mathrm{OH}$ groups of the polymer matrix involved in $\mathrm{HB}$ interactions with water. As main result, the characteristic form of the $\mathrm{HOH}$ bending mode below $270 \mathrm{~K}$, quite different from the flattened profile typically observed for polycrystalline ice, furnished experimental evidence of a liquid-like behaviour of $\mathrm{H}_{2} \mathrm{O}$ molecules inside the nanocavities of the polymer matrix. Moreover, the changes in wavenumber position and intensity upon increasing temperature are consistent with the characteristic de-structuring effect, induced by the thermal motion, on the HB network of water. Finally, and as a main conclusion, this type of temperature-related disruptive effect appears selectively triggered by the level of crosslinking density, as expressed by the molar ratio $n$.

[Please insert Figure 4 about here]

The response of the polymer backbone to the hydration and formation of a gel phase was conveniently studied by Raman spectroscopy. The analysis of the vibrational signals arising from the polymer matrix suggested that the gelation phenomena in CDNS is driven not only by the formation of $\mathrm{HB}$ networks between water and the hydrophilic groups of polymer, by also by hydrophobic hydration, such as in the establishment of non-covalent interactions between the solvent and the more hydrophobic parts of polymer skeleton [54]. The use of UV Raman scattering has the advantage of inducing a selective enhancement of the vibrational spectra the modes associated with the aromatic moieties present in the PMA-based CDNS structure [54]

The experimental UV Raman spectrum of CDNS revealed the presence of three intense signals, labelled as $v(\mathrm{C}=\mathrm{C})_{1}, v(\mathrm{C}=\mathrm{C})_{2}$, and $v(\mathrm{C}=\mathrm{O})$, respectively ascribed to a ring breathing mode, the stretching of the aromatic ring of PMA together with the bending of the $\mathrm{C}-\mathrm{H}$ groups, and the stretching of the carbonyl groups. These bands are centred at 1553, 1604 and $1721 \mathrm{~cm}^{-1}$, in the order. The observed increase in intensity of $v(\mathrm{C}=\mathrm{C})_{1}$ mode, by increasing the hydration level $h$, provided evidence that the $\mathrm{CH}$ groups of the aromatic ring of PMA are strongly involved in nonconventional $\mathrm{HB}$ interactions of the type $\mathrm{C}-\mathrm{H} \cdots \mathrm{O}-\mathrm{H}$ with engaged water molecules. This dynamic 
perturbation of the hydrophobic $\mathrm{CH}$ groups extends up to $h_{\max }=8$, then it tends to vanish probably because of the rearrangement of the $\mathrm{H}_{2} \mathrm{O}$ in tetrahedral, bulk-like environments. Further information on the interaction of the polymer backbone with the solvent came from the line-width analysis of the $v(\mathrm{C}=\mathrm{C})_{1}$ mode. The linewidth $\Gamma$ of the Raman band is related to the dephasing time $\tau_{\text {deph }}$, defined as $\tau_{\text {deph }} \cong 1 / \pi \Gamma$. The physical meaning of the dephasing time is in a reasonable estimation of the collision rate of the solvent molecules on the vibrating groups [54]. Interestingly, it was found that $\tau_{d e p h}$, after an initial increasing, becomes almost hydration-independent at the cross-over hydration level $h_{\text {cross }}$. This finding is consistent with the progressive rearrangement of water molecules to more cooperative, bulk-like networks, as a consequence of saturation sites of the water confinement of CDNS found for $h>h_{\text {cross }}$, i. e. in correspondence of the transition from a gel phase to a liquid suspension.

[Please insert Figure 5 about here]

Overall, the whole collection of the experimental results reported above suggests the possibility to modulate microscopic and macroscopic properties of CDNS by varying mainly the molar ratio $n$ during the synthesis, thus opening a facile route to the development of stimuli-responsive systems with predictable and desired drug release properties.

In this scenario of growing importance of CDNS in pharmaceutical formulations as drug container and/or carrier, a natural evolution of this research would be the clear understanding, at molecular level, of the state of the confined drug inside the polymeric network, especially in the gel state. A powerful method to assess the molecular state and the mass transport dynamics of the drug when confined in the scaffold is high resolution magic angle spinning NMR spectroscopy (HR-MAS NMR) [57]. When the drug is entrapped in viscous media - such as the hydrogels - or heterogeneous host-guest systems - such as porous polymers or nanoparticles - the NMR spectra of the drugs are heavily affected by line broadening caused by the anisotropy of magnetic susceptibility, dipolar coupling and chemical shift anisotropy. These factors can be strongly attenuated by orienting the sample at the so called "magic angle" and by spinning it in the range 1000-4000 Hz. This can be done practically by using a dedicated probe (HR-MAS probe-head) and a pneumatic unit to control the sample rotation installed on a conventional NMR spectrometer for liquid state. Thus, HR-MAS NMR allows for the use of the high resolution methods (1D-, 2D-NMR and Diffusion NMR) to investigate heterogeneous and semi-solid materials, such as nanoparticles, slurries, intact cells and tissues, soft matter. It was only recently the HR-MAS NMR techniques have been applied to study the dynamic behaviour of a drug inside a polymeric scaffold, with the aim of getting information useful for the understanding and designing the release of the drug. In this 
sense, the transport phenomena of a model drug, i.e. Ibuprofen sodium salt (IP) in $\beta$-CDEDTA nanosponges were preliminary studied by HR-MAS NMR. The use of pulsed field gradients spinecho sequences (PGSE-NMR) allowed to study the diffusivity of IP confined in the hydrogel. In particular, the experiments carried out at variable diffusion time $(\Delta)$ allowed to measure the mean square displacement (MSD) of confined IP [58] The analysis of the experimental data highlighted a non-linear relationship of MSD with the observation time, symptom of the so called "anomalous diffusion": $M S D \propto D * t^{\alpha}$, with $D=$ diffusion coefficient, $t=$ diffusion time, $\alpha=$ exponent $\neq 1$. Indeed, IP showed subdiffusive behaviour $(\alpha<1)$ in CDNSEDTA with $n=4$, and a slightly superdiffusive regime $(\alpha>1)$ in CDNSEDTA with $n=8$. The transition between a subdiffusive to a superdiffusive regime can thus be modulated by structural factors such as the cross-linking degree of the polymer. This finding provides us with a facile route to the design of the release properties if these materials. Finally, the possibility of a $\mathrm{pH}$ - and $\mathrm{T}$ dependent behaviour was also tested, in view of $\mathrm{pH}$-responsive and/or T-responsive smart materials. In this context, we addressed the issue of how the degree of H-bond association of the water molecules entrapped in the gel network and the extent of intermolecular interactions involving the hydrophobic/hydrophilic moieties of the polymer matrix cooperate to determine any $\mathrm{pH}$ and thermal activation. Once again, the main investigating tools were Raman and FT-IR spectroscopies. The spectral signals coming from the polymer matrix (particularly intense in the UV Raman spectra) and from the water solvent (strongly active in FTIRATR spectra) were analysed separately in order to provide a comprehensive view of the phenomenon [59,60]. The Raman data [59] showed that the hydrophobic moieties in the structure of NS polymers were highly sensitive to the breaking/formation of HB interactions with solvent. The dynamics of the hydrophobic moieties of the polymer skeleton were found to dominate the solvation process of the system observed at high temperatures. This could be due to the enhancement of the slight acidic behaviour of the $\mathrm{CH}$ groups on the aromatic rings coming from PMA cross-linker in the CDNS polymeric network that is triggered, in turn, by $\mathrm{pH}$ variations. This outcome suggests the importance of regulating the hydrophobic/hydrophilic balance of chemical groups in the polymer network to achieve a suitable tuning of the solvation properties in the CDNS hydrogels. The IR analysis of the $\mathrm{HOH}$ bending mode of water [60] evidenced a clear influence of the $\mathrm{pH}$ on the $\mathrm{HB}$ reorganization of confined water molecules that tend to develop an increasing number of ordered tetrahedral structures, even upon confinement, at high $\mathrm{pH}$ values. This may be due to the enhancement of hydrophilic components of the polymeric backbone and/or to the enlargement of pores in NS hydrogels occurring under basic conditions, leading to the enhancement of the swelling capability observed at macroscopic level. 
The overall results assume a particular practical importance in view of future engineering of nanosponge hydrogels for targeted delivery and release of bioactive agents.

4. Expert Opinion Cyclodextrin nanosponges (CDNS) are considered one of the most promising and innovative materials for drug delivery. They have many relevant advantages in comparison with other nanocarriers. In fact, they are recognized safe, have negligible acute or chronic toxicity, are stable in a wide range of temperature, $\mathrm{pH}$ and solvents, can be easily produced, are inexpensive and versatile as proved by dozen of applications in different fields reported in the literature. The general synthetic route is based on a single step, generally carried out under mild conditions and with high yields. Cyclodextrin nanosponges of high purity can be obtained by simple, prolonged Soxhlet extraction with suitable, non-toxic solvent such as ethanol or water. In addition, the synthetic route appears to be easily scalable. The polymeric network thus obtained is not soluble in any solvent and the resulting solid material can be easily milled up to the sub-micron scale. CDNS showed good hosting properties towards both lypophilic and hydrophilic active molecules and even towards macromolecules, proteins or enzymes. In the latter case, the effect is always to improve the enzyme stability. Also gases can be encapsulated in CDNS thus showing good adsorption and absorption capabilities. Some CDNSs also exibit high swellable properties. Neutral as well negatively charged cyclodextrin nanosponges are known. The inclusion complexes formed by using CDNS as host system always show higher stability in comparison with the corresponding complexes with parent, monomeric cyclodextrins. To date many drugs are reported to get better results when delivered by cyclodextrin nanosponges. Impressive results were in particular obtained with some anti cancer drugs namely paclitaxel and camptothecin. As nanosponges are rapidly internalized in the cells, a fast release of the drug is observed. The efficacy of the anticancer drug is enhanced up to eight folds, thus permitting the reduction of the therapeutic dose, limiting the severe side effect in chemotherapies. Nevertheless, the influence of the structure of nanosponges at molecular level in the formation of inclusion compounds and the performance of the complexes in drug delivery is still poorly explored and object of intense experimental work. The results reported in this review can significantly contribute to the rational design and optimization of new drugs/cyclodextrin nanosponges delivery systems, with particular emphasis to stimuli-responsive systems exhibiting tuneable inclusion/release properties, adapted to therapeutic demands of pathology and possible tailored carrier to selective delivery to organ or cell. In this context, cyclodextrin nanosponges represent bio-responsive nano-vehicles obtainable without high laboratory skills and complex synthetic steps. As a future perspective, and by exploiting the versatility of the syntheses, in the next years the third generation of cyclodextrin nanosponges could 
act as "stealth" nanocarriers in order to increase the circulating life-time of the drug in the fisiological environment. Additionally, positively charged nanosponges could be designed to form stable complexes with DNA, SiRNA, antisense oligonucleotides etc., thus providing innovative materials for gene delivery overcoming the present limits of non-viruses gene delivery systems such as less effectiveness in transfection and inflammatory response. It is noteworthy that "stealth" and cationic properties could be retained in a single nanosponges. Another aspect worth of investigation in the next future is the combined delivery of two or more active molecules exploiting the capability of CDNS of hosting different molecules or cations. The multi-capture properties of NS could be also exploited in order to obtain synergistic effects and to get better therapeutic response.

At the present, nanosponges are under investigation to improve the delivery of antibiotic and antiviruse drugs as well neuroprotective agents.

Finally, a deep investigation of the thermodynamic parameters associated to the establishment of host-guest interactions in solid phase in comparison to the already known data of parent cyclodextrin or their derivatives in solution will provide precious information for the design of drug delivery systems.

\section{References}

Papers of special note have been highlighted as

- of interest

" of special interest

[1] Chen G, Roy I, Yang C, Prasad PN. Nanochemistry and Nanomedicine for Nanoparticlebased Diagnostics and Therapy. Chem Rev 2016; 116: 2826-85.

"An authoritative, comprehensive review covering current status and future outlook of nanomedicine.

[2] Prasad, PN. Introduction to Nanomedicine and Nanobioengineering; Wiley-Interscience: Hoboken, NJ, 2012.

[3] Martin Del Valle EM. Cyclodextrin their uses: A review. Process Biochem 2004; 39:103346. 
-An interesting review describing the properties of cyclodextrins and their potentialities in biocatalysis, encapsulation and controlled release, as well as in many pharmaceutical applications.

[4] Kurkov SV, Loftsson T. Cyclodextrins. Int J Pharm 2013; 453(1):167-80

"A remarkable review on cyclodextrins chemistry, their complexation properties and aggregation phenomena, with particular regard to pharmaceutical applications.

[5] Duchêne D, Ponchel G, Wouessidjewe D. Cyclodextrins in targeting. Application to nanoparticles. Adv Drug Delivery Rev 1999; 36:29-40

•An interesting overview on amphiphilic cyclodextrin nanospheres as new targeting systems.

[6] Li D, Ma M. Cyclodextrin polymer separation materials. WO 9822197 (1998)

[7] Li D, Ma M. Nanosponges for water purification. Clean Technol Environ Policy 2000; 2:112-16

[8] Trotta F. Cyclodextrins in pharmaceutics, cosmetics and biomedicine: current and future industrial applications. Hoboken, NJ, USA: John Wiley \& Sons, 2011, 323-42.

[9] Ahmed RZ, Patil G, Zaheer Z. Nanosponges - a completely new nano-horizon: pharmaceutical applications and recent advances. Drug Development and Industrial Pharmacy, 2013; 39(9): 126372.

[10] Trotta F, Dianzani C, Caldera F, Mognetti B, Cavalli R. The application of nanosponges to cancer drug delivery. Expert Opin Drug Deliv 2014; 11(6): 931-41.

"A notable review focusing on cyclodextrin-based nanosponges as innovative carrier systems for the non conventional administration of anti-cancer drugs.

[11] Chilajwar SV, Pednekar PP, Jadhav KR, Gupta GJC, Kadam VJ. Cyclodextrin-based nanosponges: a propitious platform for enhancing drug delivery. Expert Opin Drug Deliv 2014; 11(1): 111-20.

"A noteworthy insight on chemical-physical properties on cyclodextrin-based nanosponges in relation to the biocompatibility, in view of a development of successful drug formulations for pharmaceutical market.

[12] Tejashri G, Amrita B, Darshana J. Cyclodextrin based nanosponges for pharmaceutical use: A review. Acta Pharm 2013; 63: 335-58.

-A deep overview of the methods of preparation, characterization techniques and main applications of cyclodextrin-based nanosponges as drug delivery systems.

[13] Trotta F, Cavalli R, Tumiatti V, Zerbinati O, Roggero C, Vallero R. Ultrasound Assisted Synthesis of Cyclodextrin Based Nanosponges, EP Pat 1786841A1, 23May, 2007.

[14] Cavalli R, Trotta F, Tumiatti W. Cyclodextrin-based nanosponges for drug delivery. J Incl 
Phenom Macrocycl Chem 2006; 56: 209-13

[15] David F. Nanosponge drug delivery system more effective than direct injection. 2010; www.physorg.com, accessed on 20.12.2011

[16] Shende P, Kulkarni YA, Gaud RS, Deshmukh K, Cavalli R, Trotta F, Caldera F. Acute and Repeated Dose Toxicity Studies of Different $\beta$-Cyclodextrin-Based Nanosponge Formulations. J Pharm Sci 2015; 104(5):1856-63

[17] Renuka S, Roderick BW, Kamla P. Evaluation of the kinetics and mechanism of drug release from Econazole Nitrate Nanosponge loaded carbapol hydrogel. Ind J Parm Edu Res 2011; 45(1):25-31.

[18] Renuka S, Kamla P. Polymeric nanosponges as an alternative carrier for improved retention of econazole nitrate onto the skin through topical hydrogel formulation. Pharm Dev Technol 2011; 16(4):367-76

[19] Gilardi G, Trotta F, Cavalli R, Ferruti P, Ranucci E, Di Nardo G, Roggero C, Tumiatti V. Cyclodextrin nanosponges as carrier for biocatalysts, and in the delivery and release of enzymes, proteins, vaccines and antibodies. WO/2009/149883 (2008)

[20] Cavalli R, Akhter AK, Bisazza A, Giustetto P, Trotta F, Vavia P. Nanosponge formulations as oxygen delivery systems. Int J Pharm 2010; 402(1-2):254-7

'An innovative work, showing the ability of cyclodextrin-based nanosponges to encapsulate, store and release oxygen for a prolonged time, so acting as a suitable carrier for gas topical delivery.

[21] Longo C, Gambara G, Espina V, Luchini A, Bishop B, Patanarut AS, Petricoin EF 3rd, Beretti F, Ferrari B, Garaci E, De Pol A, Pellacani G, Liotta LA. A novel biomarker harvesting nanotechnology identifies Bak as a candidate melanoma biomarker in serum. Exp Dermatol 2011; 20(1):29-34

[22] Guella G, Mancini I, Mariotto G, Rossi B, Viliani G. Vibrational analysis as a powerful tool in structure elucidation of polyarsenicals: a DFT-based investigation of arsenicin A. Phys Chem Chem Phys 2009; 11:2420-7

[23] Rossi B, Caponi S, Castiglione F, Corezzi S, Fontana A, Giarola M, Mariotto G, Mele A, Petrillo C, Trotta F, Viliani G. Networking properties of cyclodextrin-based cross-linked polymers probed by inelastic light-scattering experiments. J Phys Chem B 2012; 116(17):5323-7

[24] Castiglione F, Crupi V, Majolino D, Mele A, Rossi B, Trotta F, Venuti V. Inside new materials: an experimental numerical approach for the structural elucidation of nanoporous crosslinked polymers. J Phys Chem B 2012; 116(43):13133-40 
[25] Castiglione F, Crupi V, Majolino D, Mele A, Rossi B, Trotta F, Venuti V. Effect of crosslinking properties on the vibrational dynamics of cyclodextrins-based polymers: an experimentalnumerical study. J Phys Chem B 2012; 116(27):7952-8

"One of the first application of the combined use of Raman and infrared spectroscopy, supported by numerical calculations, for the structural elucidation of cyclodextrin-based nanosponges, valid, in principle, for other amorphous polymers.

[26] Crupi V, Fontana A, Giarola M, Longeville S, Majolino D, Mariotto G, Mele A, Paciaroni A, Rossi B, Trotta F, Venuti V. Vibrational density of states and elastic properties of cross-linked polymers: combining inelastic light and neutron scattering. J Phys Chem B 2014; 118(2):624-33

[27] Crupi V, Fontana A, Giarola M, Majolino D, Mariotto G, Mele A, Melone L, Punta C, Rossi B, Trotta F, Venuti V. Connection between the vibrational dynamics and the cross-linking properties in cyclodextrins-based polymers. J Raman Spectrosc 2013; 44(10):1457-62

[28] Lo Meo P, Lazzara G, Liotta L, Riela S, Noto R. Cyclodextrin-calixarene co-polymers as a new class of nanosponges. Polym Chem 2014; 5:4499 -510

[29] Ansari KA, Vavia PR, Trotta F, Cavalli R. Cyclodextrin-based nanosponges for delivery of resveratrol: in vitro characterisation, stability, cytotoxicity and permeation study. AAPS PharmSciTech 2011; 12(1):279-86

[30] Singireddy A, Subramanian S. Fabrication of cyclodextrin nanosponges for quercetin delivery: physicochemical characterization, photostability, and antioxidant effects. J Mater Sci 2014; 49(23):8140-53

[31] Castiglione F, Crupi V, Majolino D, Mele A, Panzeri W, Rossi B, Trotta F, Venuti V. Vibrational dynamics and hydrogen bond properties of $\beta$-CD nanosponges: an FTIR-ATR, Raman and solid-state NMR spectroscopic study. J Incl Phenom Macrocycl Chem 2013; 75(3-4): 247-54

[32] Mele A, Castiglione F, Malpezzi L, Ganazzoli F, Raffaini G, Trotta F, Rossi B, Fontana A, Giunchi G. HR MAS NMR, powder XRD and Raman spectroscopy study of inclusion phenomena in $\beta C D$ nanosponges. J Incl Phenom Macrocycl Chem 2011; 69(3): 403-9

[33] Taraskin SN, Loh YL, Natarajan G, Elliott SR. Origin of the Boson peak in systems with lattice disorder. Phys Rev Lett 2001; 86:1255-8

-A landmark in the study of the origin of the Boson peak in models with lattice disorder.

[34] Benassi P, Fontana A, Frizzera W, Montagna M, Mazzacurati V, Signorelli G. Disorderinduced light scattering in solids: The origin of the Boson peak in glasses. Phil Mag B 1995; $71: 761-9$

[35] Pilla O, Angelani L, Fontana A, Gonçalves JR, Ruocco G. Structural and dynamical consequences of density variation in vitreous silica. J Phys: Condens Matter 2003; 15:S995-S1005 
[36] Baldi G, Fontana A, Monaco G, Orsingher L, Rols S, Rossi F, Ruta B. Connection between Boson peak and elastic properties in silicate glasses. Phys Rev Lett 2009; 102:195502

[37] Monaco A, Chumakov A, Monaco G, Crichton WA, Meyer A, Comez L, Fioretto D, Korecki J, Ruffer R. Effect of densification on the density of vibrational states of glasses. Phys Rev Lett 2006; 97:135501

[38] Niss K, Begen B, Frick B, Ollivier J, Beraud A, Sokolov A, Novikov VN, Alba-Simionesco C. Influence of pressure on the Boson peak: stronger than elastic medium transformation. Phys Rev Lett 2007; 99:055502

[39] Hong L, Begen B, Kisliuk A, Alba-Simionesco C, Novikov VN, Sokolov AP. Pressure and density dependence of the boson peak in polymers. Phys Rev B 2008; 78:134201

[40] Caponi S, Corezzi S, Fioretto D, Fontana A, Monaco G, Rossi F. Raman-scattering measurements of the vibrational density of states of a reactive mixture during polymerization: effect on the Boson peak. Phys Rev Lett 2009; 102:027402

[41] Zanatta M, Baldi G, Caponi S, Fontana A, Gilioli E, Krish M, Masciovecchio C, Monaco G, Orsingher L, Rossi F, Ruocco G, Verbeni R. Elastic properties of permanently densified silica: a Raman, Brillouin light, and x-ray scattering study. Phys Rev B 2010; 81:212201

[42] Li J, Harada A, Kamachi M. Sol-gel transition during inclusion complex formation between $\alpha$ cyclodextrin and high molecular weight poly(ethylene glycol)s in aqueous solution. Polym J 1994; 26:1019-26

[43] Zhao SP, Zhang LM, Ma D. Supramolecular hydrogels induced rapidly by inclusion complexation of poly(e-caprolactone)-poly(ethylene glycol)-poly(e-caprolactone) block copolymers with $\alpha$-cyclodextrin in aqueous solutions. J Phys Chem B 2006; 110(25): 12225-9

[44] Li J, Ni X, Leong KW. Injectable drug-delivery systems based on supramolecular hydrogels formed by poly(ethylene oxide)s and alpha-cyclodextrin. J Biomed Mater Res A 2003; 65(2):196202

[45] Jongpaiboonkit L, Zhou Z, Ni X, Wang YZ, Li J. Self-association and micelle formation of biodegradable poly(ethylene glycol)-poly(L-lactic acid) amphiphilic di-block copolymers. J Biomater Sci Polym Edn. 2006; 17:747-763

[46] Huh KM, Cho YW, Chung H, Kwon IC, Jeong SY, Ooya T, Lee WK, Sasaki S, Yui N. Supramolecular hydrogel formation based on inclusion complexation between poly(ethylene glycol)-modified chitosan and $\alpha$-cyclodextrin. Macromol Biosci 2004; 4(2) 92-99

[47] He L, Huang J, Chen Y, Xu X, Liu L. Inclusion interaction of highly densely PEO grafted polymer brush and $\alpha$-cyclodextrin. Macromolecules 2005; 38(9):3845-51 
[48] Ren L, He L, Sun T, Dong X, Chen Y, Huang J, Wang C. Dual-responsive supramolecular hydrogels from water-soluble PEG-grafted copolymers and cyclodextrin. Macromol Biosci 2009; 9:902-10

[49] Karim AA, Loh XJ. Design of a micellized $\alpha$-cyclodextrin based supramolecular hydrogel system. Soft Matter 2015; 11:5425-34.

Very interesting paper proposing a two-step mechanism for the understanding of the formation of micellized supramolecular hydrogel systems.

[50] Crupi V, Fontana A, Majolino D, Mele A, Melone L, Punta C, Rossi B, Rossi F, Trotta F, Venuti V. Hydrogen-bond dynamics of water confined in cyclodextrin nanosponges hydrogel. J. Inclusion Phenom. Macrocyclic Chem. 2014: 80, 69-75.

[51] Castiglione F, Crupi V, Majolino D, Mele A, Melone L, Panzeri W, Punta C, Rossi B, Trotta F, Venuti V. Gel-sol evolution of cyclodextrin-based nanosponges: role of the macrocycle size. J. Inclusion Phenom. Macrocyclic Chem. 2014: 80, 77-83.

[52] Crupi V, Majolino D, Mele A, Melone L, Punta C, Rossi B, Toraldo F, Trotta F, Venuti V. Direct evidence of gel-sol transition in cyclodextrin-based hydrogel as revealed by FTIR-ATR spectroscopy. Soft Matter 2014: 10, 2320-2326.

'The first example of monitoring the phase transition from gel to liquid suspension in cyclodextrin-based hydrogels by taking into account, using FTIR-ATR technique, the connectivity pattern of $\mathrm{H}_{2} \mathrm{O}$ molecules involved in the gelation process.

[53] Crupi V, Majolino D, Mele A, Rossi B, Trotta F, Venuti V. Modelling the interplay between covalent and physical interactions in cyclodextrin-based hydrogel: effect of water confinement. Soft Matter 2013: 9, 6457-6464.

-A novel model is here proposed, aimed at understanding how physical and chemical interactions combine in determining the macroscopic properties of cyclodextrin-based hydrogel network.

[54] Rossi B, Venuti V, D’Amico F, Gessini A, Castiglione F, Mele A, Punta C, Melone L, Crupi V, Majolino D, Trotta F, Masciovecchio C. Water and polymer dynamics in a model polysaccharide hydrogel: the role of hydrophobic/hydrophilic balance. Phys. Chem. Chem. Phys. 2015: 17, 963-971.

An innovative paradigmatic model is proposed, that, making use of the complementarity of Raman and infrared spectroscopy, allows to distinguish the dynamics of water and polymer matrix in a polysaccharide hydrogel. 
[55] Rossi B, Venuti V, D’Amico F, Gessini A, Castiglione F, Mele A, Punta C, Melone L, Crupi V, Majolino D, Trotta F, Masciovecchio C. Probing the molecular connectivity of water confined in polymer hydrogels. J. Chem. Phys. 2015: 142(1), 014901, 1-1341.

[56] Venuti V, Rossi B, D’Amico F, Mele A, Punta C, Melone L, Crupi V, Majolino D, Trotta F, Gessini A, Masciovecchio C. Combining Raman and Infrared spectroscopy as a powerful tool for the structural elucidation of cyclodextrin-based hydrogels. Phys. Chem. Chem. Phys. 2015: 17, 10274-10282.

[57] Power W P. High-resolution magic angle spinning enabling applications of NMR spectroscopy to semi-solid phases. Ann. Reports on NMR Spectroscopy 2011: 72, 111-156.

"A wide comprehensive review on the evolution of the applications of HRMAS on a variety of systems ranging from polymeric samples to cells and tissues.

[58] Ferro M, Castiglione F, Punta C, Melone L, Panzeri W, Rossi B, Trotta F, Mele A. Anomalous diffusion of Ibuprofen in cyclodextrin nanosponge hydrogels: an HRMAS NMR study. Beilstein J Org Chem 2014: 10, 2715-2723.

[59] Rossi B, Venuti V, D’Amico F, Gessini A, Mele A, Punta C, Melone L, Crupi V, Majolino D, Trotta F, Masciovecchio C. Toward an understanding of the thermosensitive behaviour of $\mathrm{pH}$ responsive hydrogels based on cyclodextrins. Soft Matter 2015: 11, $5862-5871$.

•An authoritative experimental investigation, by complementary UV Raman and FTIR-ATR spectroscopies, of the molecular mechanisms responsible of the thermo-activation of pHdpendent cyclodextrin-based hydrogels.

[60] Rossi B, Venuti V, Mele A, Punta C, Melone L, D’Amico F, Gessini A, Crupi V, Majolino D, Trotta F, Masciovecchio C. Vibrational signatures of the dependence of water dynamics upon confinement in nanoporous polymer network. Phys. Chem. Chem. Phys. 2016: 18, 12252-12259. 